\title{
QoS Aware Radio Access Technology Selection Framework in Heterogeneous Networks using SDN
}

\author{
Alessandro Raschellà, Faycal Bouhafs, Deepak G. C., and Michael Mackay
}

\begin{abstract}
This paper addresses the problem of radio access technology (RAT) selection in heterogeneous networks (HetNets). Current approaches rely on signal related metrics such as signal to interference plus noise ratio (SINR) for selection of the best network for the wireless user. However, such approaches do not take into account the quality of service $(\mathrm{QoS})$ requirements of wireless users and therefore often do not connect them to the most suitable network. We propose a QoS aware RAT selection framework for HetNets based on software-defined networking (SDN). The proposed framework implements a RAT selection strategy that reflects QoS requirements of downlink flows using a metric called fittingness factor (FF). The framework relies on the flexibility and centralised nature of SDN to implement monitoring and RAT capacity assessment mechanisms that help in the realisation of the selection strategy. The simulation campaign illustrates the important gains achieved by our RAT selection framework in terms of data rates assigned to the wireless users, their satisfaction, and their quality of experience (QoE) compared against other state of the art RAT selection solutions.
\end{abstract}

Index Terms: Heterogeneous networks, potential game, radio access technology selection, and software defined networking.

\section{INTRODUCTION}

W IRELESS communication technologies have witnessed rapid progress and popularity over the last few years as the use of wireless devices and applications have grown at an immensely fast rate. However, due to the limited capacity of the radio spectrum, operators are compelled to find new ways to increase the capacity of their wireless networks and minimise spectrum congestion. In this context, the concept of heterogeneous networks (HetNets), consisting of the integration of different radio access technologies (RATs), is currently being promoted as a way to address his challenge [1].

Today, Wi-Fi and cellular networks represent the most popular RATs used in wireless data communication and will also play a key role in the evolution of HetNets in the future. Operators are increasingly deploying small cells such as pico-cells and femto-cells which, along with Wi-Fi access points (APs), will result in a spectrum densification that could increase the

Manuscript received June 9, 2017

The work in this paper is supported by the European Unions Horizon 2020 Research and Innovation Programme under Grant Agreement no. 644262 as part of the Wi-5 project.

A. Raschellà, F. Bouhafs, and M. Mackay are with the Department of Computer Science, Liverpool John Moores University, Liverpool, UK, L3 3AF. email: A.Raschella, F.Bouhafs, M.I.Mackay@ljmu.ac.uk.

D. G. C. is with the School of Computer Science and Mathematics, Kingston University, London, KT1 2EE. email: d.gc@kingston.ac.uk.

A. Raschellà is the corresponding author.

Digital Object Identifier: 10.1109/JCN.2017.000098 network capacity and will play a key role in future 5th generation (5G) technologies [2]. Unfortunately, this spectrum densification will not be sufficient to address the spectrum congestion problem and it is therefore also necessary to devise strategies that could help to manage the load among different RATs such that it could optimise the utilisation of the spectrum resources.

On the other hand, many of the applications used on today's wireless devices have different quality of service $(\mathrm{QoS})$ and quality of experience (QoE) requirements. For instance, users can run applications such as WhatsApp, Instagram, and Viber to share pictures, videos, and establish voice conversations from their phones. They can also use tablets and smartphones as a second screen or for online video gaming, which again have different QoS requirements than other applications. Such differentiation in QoS and QoE requirements is currently not reflected in the allocation and management of spectrum resources in multiRATs environments. Currently, wireless users connected to long term evolution (LTE) networks are usually offloaded towards unlicensed Wi-Fi spectrum only if the signal to interference plus noise ratio (SINR) is below a certain threshold established in a LTE connection. Hence, following the standard behind LTE/WiFi network selection, the best connectivity still corresponds to the best provided SINR [2], [3]. Furthermore, in the specific case of Wi-Fi, the AP selection approach for wireless users is usually based on the best received signal strength (RSS) as recommended by the IEEE 802.11 standard [4].

More recently, software-defined networking (SDN) [5] has emerged as an open, efficient and flexible network management concept for large networks. By decoupling the control plane from the data plane, SDN can centralise network management operations in a single entity, often referred to as a controller. This centralized management approach allows us to program large networks through the OpenFlow protocol [6]. Projects such as EmPOWER [7] and Wi-5 [8] have already developed Wi-Fi network management frameworks based on Wireless SDN.

In this paper we propose a QoS aware RAT selection framework which relies on SDN to efficiently provide optimised connectivity for applications to a specific RAT, matching them with the most suitable access network node considering their QoS requirements. This framework is based on the SDN architecture designed and implemented in the context of the H2020 Wi-5 (What to do With the Wi-Fi Wild West) project [8], which has been developed to address spectrum congestion in Wi-Fi networks. The first version of this architecture has been designed and implemented to address radio resource management (RRM) strategies and horizontal handover in wireless local area networks (WLANs) [9], [10], and its extension that will also allow vertical handover between Wi-Fi and $3 \mathrm{G} / 4 \mathrm{G}$ mobile networks is 
currently in progress.

The rest of the paper is organized as follows: in Section II we provide a comprehensive analysis of the state of the art in the context of RAT selection solutions and introduction of Wireless SDN for RRM in HetNets, illustrating our main scientific contributions. In Section III, we present our RAT selection framework, highlighting the benefits of using SDN for an efficient implementation of RAT selection policy. In Section IV, we provide the analytical formulation that models the proposed RAT selection strategy, which is then presented in Section V. In Section VI, we illustrate the simulation model we used to assess our RAT selection solution together with the evaluation results. Finally, Section VII presents our concluding remarks and future works.

\section{STATE OF THE ART AND PAPER CONTRIBUTIONS}

In the existing literature, many studies focus on the development of RAT selection solutions in HetNets such as [2]-[4], and [11]-[20]. RAT selection approaches can be classified as either distributed [2], [4], [11], [14], and [15], or centralised [3], [12], [13], [16]-[20] approaches. With distributed solutions, a wireless device usually gathers performance related measurements from the network before selecting the most suitable RAT according to a specific metric while centralised approaches rely on the global view obtained from the network controller to decide the best RAT. There are also further different ways to classify the works in this area. For instance, papers [4], [11], [13], and [20] focus only on offloading the traffic from LTE to Wi-Fi networks. Other studies focus on RAT selection solutions for HetNets involving only LTE cells such as macro, femto and picocells [12], [17], and [18]. Finally, the works in [2], [3], [14][16], and [19] propose a complete integration between LTE and Wi-Fi going beyond the mere offloading from one network to another.

Moreover, in the domain of RRM in HetNets based on SDN technology, several innovative solutions have recently been proposed in the literature. For instance, the authors in [18] propose the use of SDN to handle all the control information among the network elements to address unexpected back-haul failures in 4G/5G HetNets, but without the inclusion of Wi-Fi technology. In [19] the authors propose a RAT scheme based on SDN where the users connect to the access nodes with the channel capacity that meets the bandwidth requirements of their applications. While in [20], an SDN-based offloading control mechanism is proposed to orchestrate the offloading from LTE femto-cells to Wi-Fi nodes according to a users' dissatisfaction parameter. Finally, in [21] and [22] the authors propose the design of architectures based on SDN, illustrating the benefits achievable through its use in terms of spectrum management in the context of the 5G. On the other hand, these works do not address RAT selection.

The most important novelties and contributions of this paper with respect to the above mentioned state-of-the-art can be summarised as follows:

- We propose a novel strategy that matches the most suitable RAT for a certain user based on QoS requirements for his/her ongoing application. Such a match will allow smart use of the limited spectrum resources guaranteeing the users' QoS demands in the most efficient way. The SDN controller provides all the monitoring information needed for our RAT selection strategy to allow a complete and efficient integration between LTE and Wi-Fi technologies.

- We propose a RAT Selection Framework based on SDN that allows the implementation of the proposed strategy. Specifically, this framework exploits the capabilities offered by SDN including cross-layer monitoring and centralised management of different networks which enables seamless handover, thus allowing the implementation of an efficient RAT selection strategy. In this framework, the SDN controller is able to obtain monitoring information about the status of the network and execute relevant algorithms to react accordingly while respecting the requirements of the wireless users. We have simulated the framework and assessed its performance in terms of QoS and QoE requirements satisfaction.

\section{RAT SELECTION FRAMEWORK}

The RAT selection framework proposed in this work exploits the flexibility and centralised nature of SDNs where the controller is able to take into account the QoS requirements of wireless users and manage access to RATs accordingly. In this approach, the SDN controller is able to match and then select the best RAT for each downlink flow. The use of SDN in the proposed framework allows for a single and global point where all the control operations can be integrated. To better highlight the benefits of using SDN to implement an efficient and QoS aware RAT selection approach, we consider the scenario depicted in Fig. 1. In this scenario, wireless users with different applications and QoS requirements need to connect to the radio access network (RAN) that could satisfy them best. Specifically, the left side of the figure represents the RAT selection based on 3rd generation partnership project (3GPP) and 802.11 standards, i.e., each device is connected to the node providing a SINR which is above the predefined thresholds. On the other hand, this standard approach does not take into consideration the possible congestion of a certain node. For instance, the left side of the figure shows how users watching videos requiring high data rates but the capacity of their AP (i.e., AP1 in the figure) and do not allow satisfactory connection to the user trying to make a call (i.e., User3 in the figure). While, the right side of the figure illustrates the benefits achievable through our approach based on SDN. In detail, the SDN controller detects and selects the LTE node (i.e., HeNB1 in the figure) as the most suitable for User3 because this node is able to provide a better connection for the call, avoiding the congestion around AP1 (despite it having the lowest SINR), and satisfying all the users connected to the networks under its control.

\section{A. Wi-5 SDN Architecture}

The SDN framework used to implement our RAT selection approach is based on the architecture presented in [9] which was developed in the context of the $\mathrm{H} 2020 \mathrm{Wi}-5$ project [8], and is illustrated in Fig. 2. In this architecture, management solutions are implemented as applications on the northbound API of the SDN controller and algorithms for AP selection [23], [24], chan- 


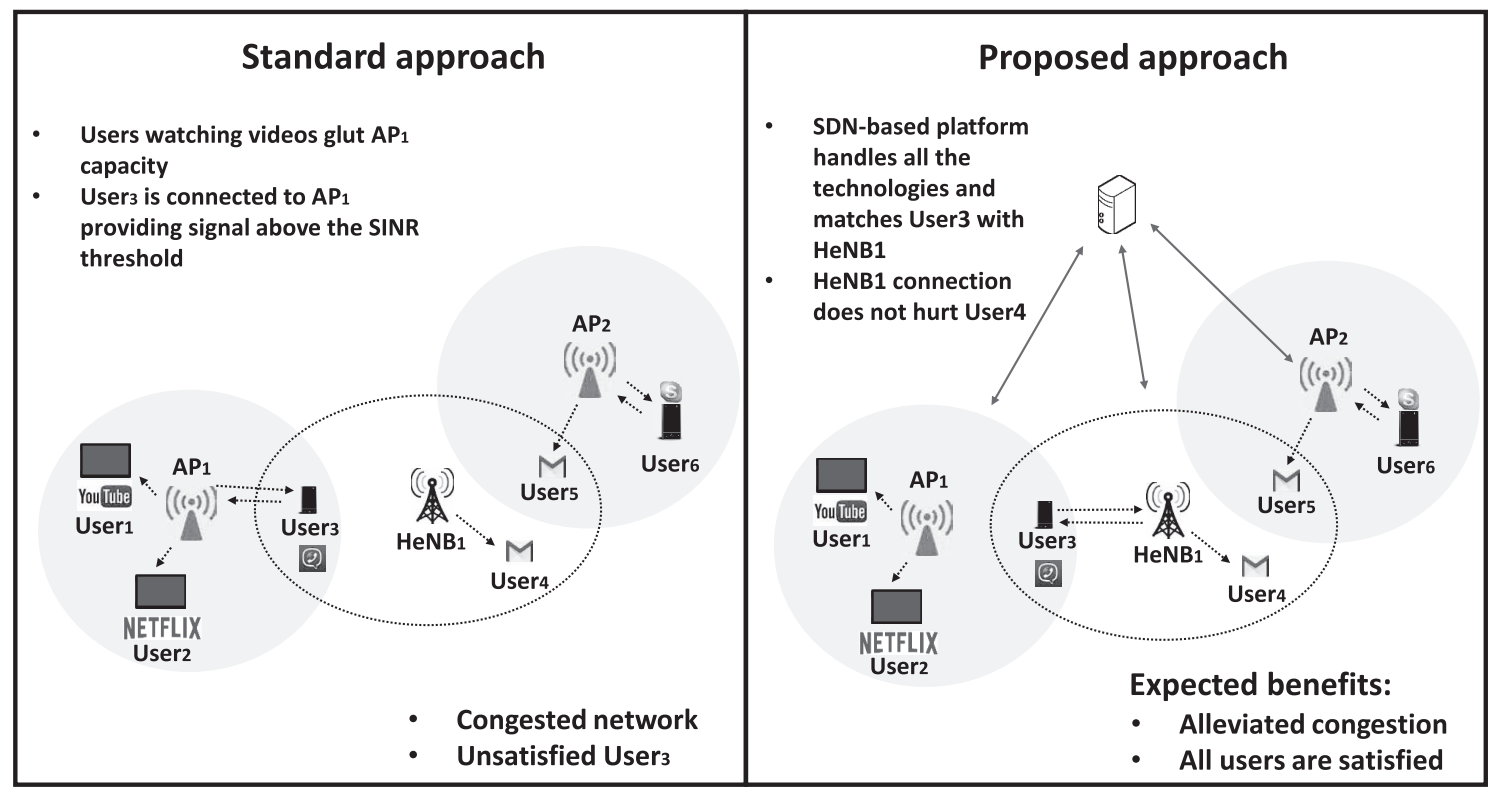

Fig. 1. Example of RAT selection implementation using SDN.

nel assignment [25], [26], and RRM [27] have been already proposed for implementation to address spectrum congestion in WiFi networks. The Wi-5 architecture defines a Spectrum Plane which enhances the operational capabilities of IEEE 802.11 APs by defining new monitoring and configuration primitives, and making APs programmable, thereby enabling fine-grained spectrum allocation and management. As illustrated on the right side of Fig. 2, this plane is in addition to the data plane that is part of traditional SDN architectures, where data traffic management policies reside. The Spectrum Plane also provides an implementation of dynamic channel assignment (DCA), transmit power control (TPC), and a monitoring function that measures the interference level and the load in each channel [10]. The monitoring function of the Spectrum Plane keeps track of the number of clients associated with each AP, the amount of traffic and its nature. Hence, this capability allows it to determine the QoS requirements of the traffic each station is sending and receiving, and implement intra-AP power adjustments according to the requirements of each flow.

\section{B. SDN-Based Framework for RAT Selection}

Many research efforts, including Wi-5, are currently attempting to support the management of cellular networks in order to ease spectrum congestion. This requires extending the southbound API of the SDN controller to be able to configure the parameters of these networks and their access nodes. This is particularly helpful in the context where a single operator manages both RANs: cellular and Wi-Fi. In such situation, the operator can use the SDN controller to manage access to both networks and assist wireless users with their QoS demands. Such a vision is already being promoted as part of $5 \mathrm{G}$, where operators are expected to manage heterogeneous networks consisting of several RATs [1], [2]. Building on this latest development, we consider the scenario of a HetNet in which the RANs include a set $N$ of $n$ wireless technologies tightly merged in a unique wireless ac- cess network under centralised SDN-based control. Specifically, RATs include Wi-Fi APs, femtocell LTE base stations (HeNB) and macrocell LTE base stations (eNodeBs). The controller is able to handle all the access nodes of its HetNet and provide connection to a set $M$ of $m$ application flows required by wireless users trying to connect to the network. Note that each flow can be either a flow for applications required by a Wi-Fi station (STA), or by a dual-interface device (e.g., smartphone, tablet, etc.) connected by Wi-Fi or LTE technology.

When receiving each station connection Request message redirected from the RAN, the SDN controller triggers the RAT selection policy running on the controller as illustrated in Fig. 3. Specifically, for each new flow trying to connect to the network, the controller finds the optimized node allocation for all the application flows active in the network. All the details on the execution of the RAT selection policy will be explained in Section IV and Section V. We, therefore, define the following modules depicted in Fig. 3, upon which our solution relies to achieve this dynamic RAT selection strategy: Provided quality assessment (PQA), required quality assessment (RQA) and decision making (DM).

The PQA module gives information on the bit rate that each accessible node of the network can achieve for a new station request, measured at the physical layer connection. The assessment is obtained by the computation of the link capacity available for each new flow in terms of the bit rate, which in turns depends on the monitoring information received by the controller through the monitoring function, such as the channel bandwidth assigned to each node, the measured inter-nodes interference within the network, and the position of the station requiring the connection. The details of this computation will be provided in the next section.

The RQA module translates the QoS requirements of a connection-requesting station achieved through the monitoring function into a bit-rate metric. The QoS requirements of the 


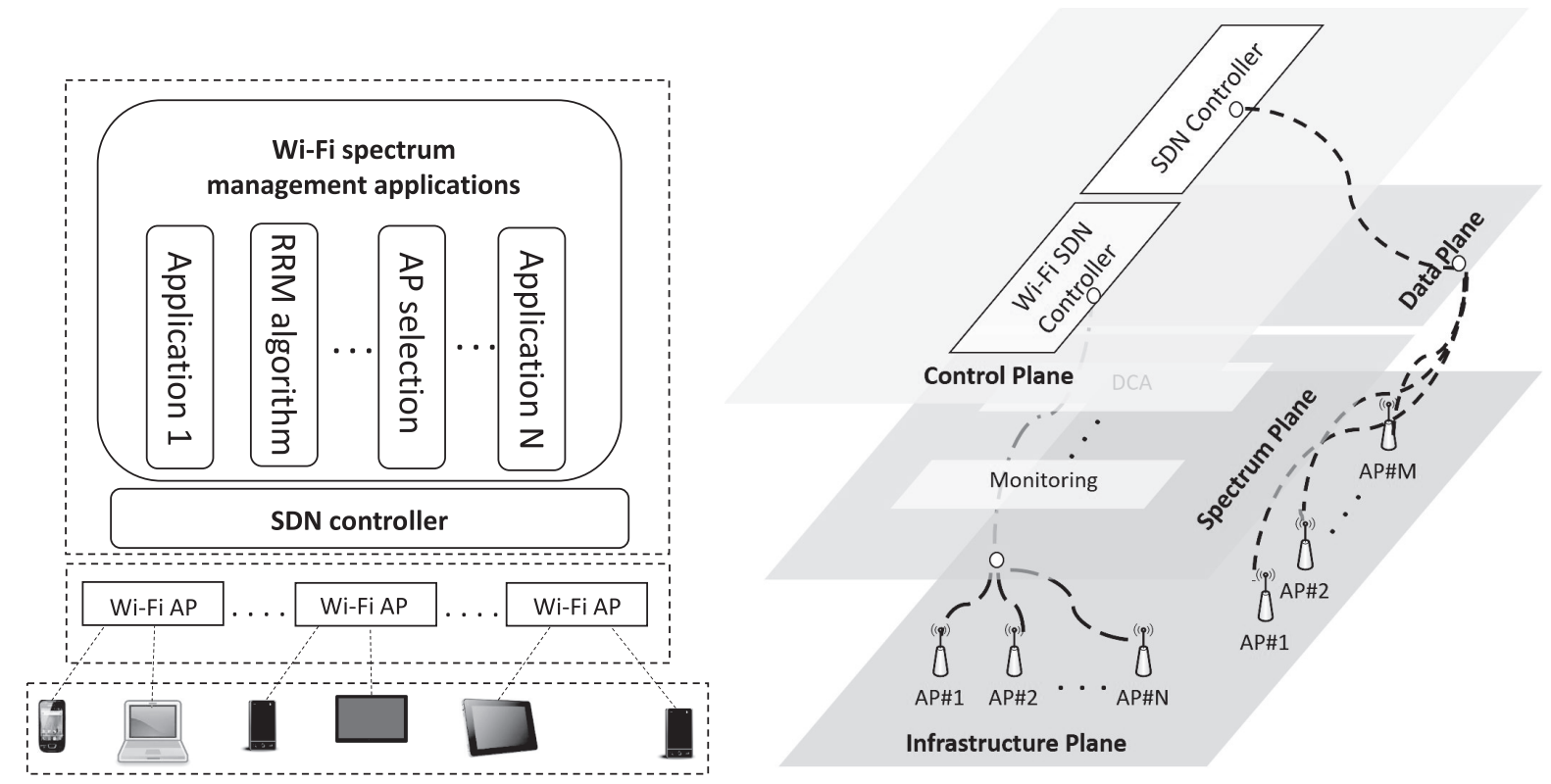

Fig. 2. Wi-5 architecture for Wi-Fi networks nanagement.

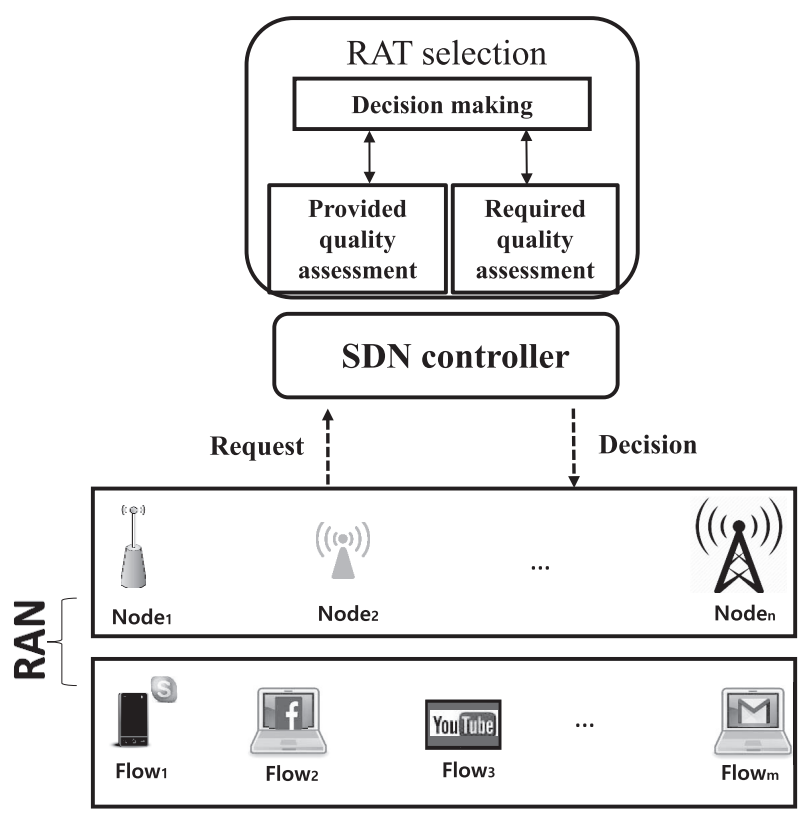

Fig. 3. SDN-based framework for RAT selection.

station depend on the nature of the data flow that the station is sending and receiving. These QoS requirements can easily be either proactively programmed into the SDN controller [28], or reactively inferred through QoS detection techniques such as machine learning (ML) strategies. In particular, the application of ML strategies to detect traffic in real-time has attracted significant attention in past works [29], [30]. For example, the MLbased classification approach presented in [30] achieves 99\% classification accuracy for voice over IP (VoIP) traffic across the nodes of their network. Therefore, this capability can be easily implemented to work in our framework but the details of such an implementation are outside the scope of this paper. Hence, we assume that the information used by this process to compute the QoS requirements is available.

The DM module is triggered every time a new flow $i$ needs to be associated to a node $j$. It first collects the available information from the PQA and RQA modules, which depends on the radio environment. These information are the available bit rate and the required bit rate for all the flows active in the network. Then, it uses this information to efficiently match the most suitable available bit rates provided by the nodes for the required bit rates. The analytical details about the matching process developed in the DM and its role in the execution of the RAT selection is explained in the next sections.

\section{PROBLEM FORMULATION}

In order to model the proposed RAT selection problem implemented in the DM module, we need to firstly define the fittingness factor (FF) metric. This metric, which has been introduced in [31], depends on the available bit rate and on the required bit rate, provided by PQA and RQA, respectively. It thus helps to associate the downlink flow's device to the most suitable RAT. Therefore, this section provides a comprehensive description of the computation of the available bit rate in each RAT and the definition of the FF.

\section{A. Available Bit Rate}

In our RAT selection strategy, after receiving the Request from the RAN, the PQA is able to compute the available bit rate in each accessible RAT for the new flow. The available bit rate for a generic flow $i$ in a generic node $j, b_{i, j}$, is computed depending on each specific RAT. In detail, the values of the SINR experienced by a certain flow in any accessible RAT is computed at the location of the user requiring connection for the flow as follows: 


$$
S I N R_{i, j}=\frac{g_{i, j} \cdot p_{j}}{\sum_{k \in N^{\prime}} g_{i, k} \cdot p_{k}+N_{0}} .
$$

Here, $g_{i, j}$ is the channel gain from node $j$ to flow $i, p_{j}$ is the transmit power of node $j, N_{0}$ is the additive Gaussian white noise, and $N^{\prime} \subseteq N$ is the set of nodes interfering with node $j$ and therefore, affecting the SINR experienced by flow $i$. The computation of the link capacity $b_{i, j}$ between flow $i$ and node $j$ for each technology included in the RAN is explained below.

In the case of Wi-Fi, the link capacity of a node corresponds to the most efficient modulation and coding scheme (MCS) to achieve the highest available bit rate under the interference level constraints. Moreover, we consider the MCSs computed by using the orthogonal frequency division multiple access (OFDMA) approach, which has been adopted in most 802.11 protocols (e.g., $802.11 \mathrm{~g} / \mathrm{a} / \mathrm{n}$ ).

Specifically, according to the $802.11 \mathrm{~g} / \mathrm{a} / \mathrm{n}$ standards, there exists a set of defined bit rate levels included between $1 \mathrm{Mbps}$ and $54 \mathrm{Mbps}$ that can be provided by the nodes. Each of these bit rate levels represents the maximum link capacity in $\mathrm{Wi}-\mathrm{Fi}$ APs, $b_{i, j}^{W F}$, between flow $i$ and AP $j$ that can be computed using $S I N R_{i, j}$ and $B W_{j}$, which is the bandwidth assigned to AP $j$ in $\mathrm{Hz}$, through the Shannon-Hartley theorem [24]. In detail, first the parameter $b_{i, j}^{W F^{\prime}}$ is computed by (2) and then, $b_{i, j}^{W F}$ is achieved by mapping $b_{i, j}^{W F^{\prime}}$ to the closest bit rate level allowed by OFDMA.

$$
b_{i, j}^{W F^{\prime}}=B W_{j} \cdot \log _{2}\left(1+S I N R_{i, j}\right)
$$

In the case of LTE, the SINR measured at the location of a user requiring connection is mapped to the corresponding channel quality indicator (CQI), which represents the highest possible MCS that the user's device can process with a block error rate lower than $10 \%$ [32], [33]. In LTE systems, 15 different CQI levels illustrated in Table 1 are foreseen. The LTE air interface uses OFDMA in the downlink direction and the available sub-carriers are grouped into resource blocks (RBs). Each RB is a sub-channel of capacity $C_{R B}$ equal to $180 \mathrm{kHz}$ and formed by 12 consecutive and equally spaced sub-carriers, each one lasting $0.5 \mathrm{~ms}$ [34]. The total number of available RBs at node $j, N_{R B j}$, depends on the bandwidth assigned to node $j, B W_{j}$, and allows us to compute the maximum link capacity in LTE base stations (BSs), $b_{i, j}^{L T E}$, for flow $i$ experiencing $C Q I_{i}$. Therefore, considering $S E_{i}$ as the spectral efficiency which corresponds to $C Q I_{i}$ and shown in Table 1 , and $N_{R B j}$ defined through the assigned $B W_{j}, b_{i, j}^{L T E}$ can be expressed by (3):

$$
b_{i, j}^{L T E}=S E_{i} \cdot C_{R B} \cdot N_{R B j} .
$$

After the computation of $b_{i, j}^{W F}$ and $b_{i, j}^{L T E}$ provided by the PQA, the DM also computes the bit rate that can be served to flow $i$ by node $j$ called here $R_{i, j}$, through the resource allocation algorithm defined in [24]. Note that this value also depends on the number $M_{j}$ of all other flows connected to node $j$, and the maximum capacity $C_{j}$ in bps available in node $j$ and then, it can be expressed as a function of all these parameters:

$$
R_{i, j}= \begin{cases}\Phi^{W F}\left(b_{i, j}^{W F}, M_{j}, C_{j}\right), & \text { :in case of AP } \\ \Phi^{L T E}\left(b_{i, j}^{L T E}, M_{j}, C_{j}\right), & \text { :in case of BS }\end{cases}
$$

Table 1. CQI-MCS mapping.

\begin{tabular}{|c|c|c|c|}
\hline $\begin{array}{c}\text { CQI } \\
\text { Index }\end{array}$ & $\begin{array}{c}\text { Modulation } \\
\text { Scheme }\end{array}$ & $\begin{array}{c}\text { Code } \\
\text { Rate }\end{array}$ & $\begin{array}{c}\text { Spectral } \\
\text { Efficiency } \\
\text { (bits/s/Hz) }\end{array}$ \\
\hline 1 & QPSK & 0.076 & 0.1523 \\
\hline 2 & QPSK & 0.120 & 0.2344 \\
\hline 3 & QPSK & 0.190 & 0.3770 \\
\hline 4 & QPSK & 0.300 & 0.6016 \\
\hline 5 & QPSK & 0.440 & 0.8770 \\
\hline 6 & QPSK & 0.590 & 1.1758 \\
\hline 7 & $16-$ QAM & 0.370 & 1.4766 \\
\hline 8 & $16-$ QAM & 0.480 & 1.9141 \\
\hline 9 & $16-$ QAM & 0.600 & 2.4063 \\
\hline 10 & $16-$ QAM & 0.450 & 2.7305 \\
\hline 11 & $16-$ QAM & 0.550 & 3.3223 \\
\hline 12 & $16-$ QAM & 0.650 & 3.9023 \\
\hline 13 & $16-$ QAM & 0.750 & 4.5234 \\
\hline 14 & $16-$ QAM & 0.850 & 5.1152 \\
\hline 15 & 16-QAM & 0.930 & 5.5547 \\
\hline
\end{tabular}

Further details on this computation can be found in [24].

\section{B. Fittingness Factor}

The matching between the flow and the node is computed through the so-called FF parameter. From a general perspective, we formulate the FF parameter for flow $i$ and node $j, f_{i, j}$, by extending the concept of the sigmoid function $\Omega_{i, j}$, which is typically used to denote the bit rate achievable by a certain user $i$ from an access node $j$ for the requested bit rate [35]. Note that with the sigmoid-based utility function, the value of $\Omega_{i, j}$ increases as the bit rate for serving flow $i$ by node $j$ increases with respect to the bit rate required for flow $i$. The aim of the proposed FF is to target a more efficient association to a node through the FF concept by penalising this value if the bit rate for serving flow $i$ by node $j$ is much larger than the bit rate required for flow $i$ in order to address the suitability of a node for a flow in terms of its available bit rate. The FF metric computation considered in this paper is based on the formulation defined in [24], [36]. Specifically, for each flow $i$ and each node $j$, a FF metric is calculated as follows:

$$
f_{i, j}=\frac{1-e^{-\frac{\Omega_{i, j}}{\rho \cdot\left(R_{i, j} / R_{r e q, i}\right)}}}{\lambda} .
$$

Here $R_{\text {req }, i}$ denotes the bit rate required for flow $i ; R_{i, j}$ denotes the bit rate served to flow $i$ by node $j$. Note that $R_{r e q, i}$ is obtained via the RQA module and $R_{i, j}$ is computed in the DM module through the information obtained via the PQA and (4). While $\Omega_{i, j}$ denotes the mentioned utility function, defined by the following formula:

$$
\Omega_{i, j}=\frac{\left[\rho \cdot\left(R_{i, j} / R_{r e q, i}\right)\right]^{\zeta}}{1+\left[\rho \cdot\left(R_{i, j} / R_{r e q, i}\right)\right]^{\zeta}} .
$$

The parameters $\zeta$ and $\rho$ reflect the different degrees of elasticity between the required bit rate and the bit rate available in 


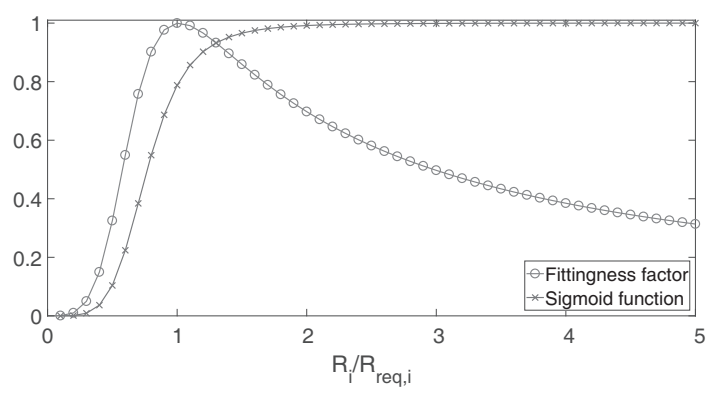

Fig. 4. Fittingness factor vs. sigmoid function.

the node, and $\lambda$ in (5) is a normalization factor used to ensure that the FF metric does not exceed 1 and expressed by (7):

$$
\lambda=1-e^{-\frac{1}{(\zeta-1)^{1 / \zeta}+(\zeta-1)^{(1-\zeta) / \zeta}}}
$$

A detailed analysis on the effect of changing the $\zeta$ and $\rho$ parameters on the FF behaviour can be found in [24]. Fig. 4 plots the evolution of the FF and the sigmoid function $\Omega$ computed through (5) and (6), respectively, as a function of the ratio between the available bit rate served to a certain flow $i$ and its required bit rate. In this example we have selected $\zeta=5$ in (6) and (7) because this value allows a smooth decrease of the FF when the available bit rates gradually become larger than the requirement [24]. Moreover, in this figure we have selected $\rho=1.3$ in (5) and (6), which allows us to reach the maximum value of the FF when the assignment equals the requirement (i.e., when $R_{i} / R_{r e q, i}=1$ ). Through this figure we aim to illustrate the difference between the behaviours of the FF and a typical sigmoid function. In detail, from the figure we can notice that the FF allows us to maximize the more suitable assignment (i.e., the case when the assignment corresponds to the requirement) rather than the highest one like the sigmoid function.

\section{RAT SELECTION STRATEGY}

The RAT selection strategy proposed in this paper is based on a potential game, which allows an efficient distribution of the wireless users among the nodes of the network handled by the SDN controller, as illustrated in Fig. 3. Potential games are a tool that allows us to perform a distributed optimization of the resource allocation through the convergence to a pure Nash equilibrium (NE), which is always guaranteed [37], [38]. The main drawback of this tool is the complexity resulting from its implementation in large distributed scenarios such as enterprise wireless networks; in fact, players usually require overall information about the remaining players of the network, making the solution not scalable. On the other hand, our framework based on SDN allows us to store all the required information on the controller, so the game can be played at this central control entity exploiting its potentiality and overcoming its drawbacks.

Hence, for each new flow trying to connect to the network, the controller plays a potential game for all the flows active in the network, to find the optimized node allocation for all of them. Specifically, in order to optimize the distribution of the $m$ flows to be served by the $n$ nodes of the network, we consider the net- work utility function $U$ as the $\log$-sum of the FFs of all the $m$ flows connected to the network. We therefore aim to optimize, through $U$, the sum of the logarithms of the FFs provided by the nodes allocated to each flow $i$ connected to its corresponding node, node $_{i}$, in order to guarantee a proportional fairness in the node allocations. On the other hand, in the considered scenario, any flow might achieve an FF value equal to zero. Therefore, in order to avoid a possible inclusion of zero in the logarithm argument, we consider a modified version of the objective function, with the sum of the logarithms of the FFs plus one [38]. Therefore, $U$ to be optimized can be defined as follows:

$$
U=\sum_{i=1}^{m} \log \left(f_{i, \text { node }_{i}}+1\right) .
$$

With this definition, it can be demonstrated that if the controller improves the utility function for only one player given the most recent action made for the other players, then the process will always converge in finite steps to an NE [38]. Each time a new flow needs to connect to the network, the RAN triggers the RAT selection strategy, which is executed through the modules implemented in the SDN controller and illustrated in Fig. 3, using the following tasks:

- Task 1: The DM collects from the RQA all the bit rates required by the flows active in the network.

- Task 2: The DM collects from the PQA all the link capacities in terms of the bit rate, which each node $j$ can provide to each flow $i, b_{i, j}^{W F}$ and $b_{i, j}^{L T E}$, using (2) in the case of Wi-Fi-based nodes and (3) in the case of LTE-based nodes.

- Task 3: The DM starts a sequential game with round robin scheduling to find the optimized value of $U$ through (8) until the pure NE is found. Specifically, in each round, for each flow $i$ connected to the network and for each node $j$ covering the area in which flow $i$ takes place, the DM first updates all the FFs of the flows affected by the connection of flow $i$ in node $j$ through (4)-(6) and then it computes $U$ that needs to be optimized, including such updated FFs, $f_{i, \text { node }_{i}}$. Note that the optimization of the log-sum takes into consideration the interest of all the $m$ flows connected to the network. The $\mathrm{NE}$ is found when the controller does not further improve the utility $U$. The analytical details of the converged NE implemented in the proposed RAT selection strategy are out of the scope of this paper and can be found in [38].

The time complexity of the game played by the controller in Task 3 is related to the following factors: 1) The number of rounds $r$ required to reach the NE; 2) the number of steps at each round that, considering the use of a round robin strategy, corresponds to the number of flows $m$; and 3) the number of nodes that on average provide coverage for a flow included in the network and defined as $\bar{n}$. Note that the number of nodes, which provide coverage to a certain flow is always lower than $n$, so $n<\bar{n}$. Therefore, the time complexity of our RAT selection strategy is linearly related to the number of flows and we can define its approximation as $O(r \cdot m \cdot \bar{n})$. 
Table 2. Simulation parameters

\begin{tabular}{|c|c|c|}
\hline Parameter & Wi-Fi AP & LTE HeNB \\
\hline $\begin{array}{c}\text { Operating } \\
\text { Frequencies }\end{array}$ & $\begin{array}{c}2.412- \\
2.472 \mathrm{GHz}\end{array}$ & $2100 \mathrm{MHz}$ \\
\hline $\begin{array}{c}\text { Channel } \\
\text { Bandwidth }\end{array}$ & \multicolumn{2}{|c|}{$20 \mathrm{MHz}$} \\
\hline Transmit Power & \multicolumn{2}{|c|}{$20 \mathrm{dBm}$} \\
\hline $\begin{array}{c}\text { Maximum } \\
\text { Capacity }\end{array}$ & $54 \mathrm{Mbps}$ & $100 \mathrm{Mbps}$ \\
\hline Node Gain & $2 \mathrm{dBi}$ & $2.2 \mathrm{dBi}$ \\
\hline Path Loss & Log-distance model \\
\hline Noise Power & \multicolumn{2}{|c|}{$-92 \mathrm{dBm}$} \\
\hline
\end{tabular}

\section{EVALUATION AND RESULTS}

\section{A. Evaluation Scenario and Metrics}

In order to evaluate our proposed RAT selection framework, we simulate a HetNet managed by an SDN controller. In addition to the controller, the HetNet consists of 20 nodes that include $15 \mathrm{Wi}$-Fi APs and 5 LTE HeNB. These nodes are randomly deployed in an area of $250 \times 250 \mathrm{~m}^{2}$ at a minimum distance of $40 \mathrm{~m}$ among them. This distribution of nodes represents a realistic and typical example of a dense environment with overlapped coverage areas among the nodes [27], [39]. We also simulate a set of $m$ downlink flows requesting connection, where $m$ varies between 1 and 400 . In order to reflect the heterogeneity of radio access in these simulated flows we assume the following:

- Single-RAT flows (SRFs) that are related to wireless devices that can only connect to a Wi-Fi AP. These flows represent $10 \%$ of the overall flows generated in the network.

- Multi-RAT flows (MRFs) that are related to wireless devices that can connect to a Wi-Fi AP and an LTE HeNB in overlapped areas.

A performance analysis is provided for all the flows, i.e., SRFs and MRFs, connecting to the network and managed by the SDN controller. Other simulation parameters that help to define a typical dense HetNet scenario such as the propagation and node settings are included in Table 2 [27], [39], [40].

In order to benchmark the performance of the proposed RAT selection framework, we compare it against the following reference strategies:

1. RAT selection scheme based on 3GPP and 802.11 standards.

Here, in case of SRFs, a flow is associated to the Wi-Fi AP providing the highest RSSI. While in case of MRFs, the WiFi-preferred scheme, which is typical in dense urban environments, is considered. Specifically, in areas where Wi-Fi and LTE are both available a MRF is associated to the AP providing the highest SINR if it is above a threshold equal to $3 \mathrm{~dB}$, otherwise to the HeNB [3], [11].

2. RAT selection load-aware scheme proposed in [3], which assigns each flow to a RAT based on the best throughput estimation. We consider this load-aware scheme because it also targets a similar approach which relies on a network-based centralised scheme for the RAT assignment. By comparing our solution to this scheme, we demonstrate that the monitor- ing information available at the SDN-based controller allow us to compute the FF, which address the suitability concept achieving better performance against such an RAT selection strategy.

The evaluation of our approach against the above two strategies focuses on the following performance metrics:

- Average Data Bit Rate: This is the statistical distribution of the data rates assigned to all the flows (e.g., minimum, maximum and median values).

- Satisfaction Percentage: This is the percentage of flows connected to one of the RANs that are served with bit rates that are higher than or equal to their given requirements, and updated for each new connection.

- Percentage of Flows with Good Mean Opinion Score (MOS): This metric is considered to address the QoE of an application provided to a certain flow as the perceived acceptability from the user's perspective [41]. In this paper we use the MOS as a metric that reflects the user's view on the quality of the network. The MOS is an arithmetic mean of all the individual scores obtained by the result of subjective tests, which can range from 1 (worst) to 5 (best). The meaning of each score is shown in Table 3 in terms of quality and impairment. In the context of our analysis, we illustrate the percentage of flows that obtain at least a Good quality at the end of the simulation.

Note that the QoS requirements of the active flows from devices trying to connect have been randomly generated from a set of bit rates that range between $40 \mathrm{kbps}$ and $5 \mathrm{Mbps}$. We have considered these values in order to represent most common online applications such as VoIP, Video Streaming, etc. which are summarised in Table 4. Specifically, for each application in this table, we illustrate: (i) The bit rate requirements, (ii) the achievable MOS when assigning these requirements, (iii) the corresponding quality perceptible by the end-user, and (iv) the impairment corresponding to the quality.

In the case of VoIP, we have considered $40 \mathrm{kbps}$ and $50 \mathrm{kbps}$, which are the approximate bit rate requirements that guarantee a Good MOS when the G.729 codec and G.726 codec are used, respectively ${ }^{1}$. While in the case of video streaming, the minimum bit rate requirement for watching videos on YouTube is $500 \mathrm{kbps}$, and it is $1 \mathrm{Mbps}$ in the case of premium content such as movies, TV shows and live events ${ }^{2}$; and finally, 5 Mbps is the minimum bit rate recommended for high definition (HD) quality videos on Netflix ${ }^{3}$. A detailed analysis that explains the relation between the Good MOS and the guaranteed minimum bit rate requirements illustrated in Table 4 can be found in [24].

Note that, for the sake of simplicity, in the analysis of the performance we illustrate the achieved results only for downlink transmissions also in case of VoIP. This is a reasonable assumption, since maintaining the minimum bit rates required for VoIP illustrated in Table 4 guarantees the Good MOS for both downlink and uplink transmissions.

\footnotetext{
${ }^{1}$ http://www.cisco.com/c/en/us/support/docs/voice/voice-quality/7934bwidth-consume.html (accessed June 2017).

${ }^{2}$ https://support.google.com/youtube/answer/78358?hl=en-GB (accessed June 2017).

${ }^{3}$ https://help.netflix.com/en/node/306 (accessed June 2017).
} 
Table 3. Mean opinion score (MOS).

\begin{tabular}{|c|c|c|}
\hline MOS & Quality & Impairment \\
\hline 5 & Excellent & Imperceptible \\
\hline 4 & Good & $\begin{array}{c}\text { Perceptible but not } \\
\text { annoying }\end{array}$ \\
\hline 3 & Fair & Slightly annoying \\
\hline 2 & Poor & Annoying \\
\hline 1 & Bad & Very annoying \\
\hline
\end{tabular}

Table 4. Bit rate requirements and MOS.

\begin{tabular}{|c|c|c|c|c|}
\hline Application & Bit rate & MOS & Quality & Impairment \\
\hline VoIP G.729 & $40 \mathrm{kbps}$ & 3.92 & & \\
\hline VoIP G.726 & $50 \mathrm{kbps}$ & 3.85 & & \\
\hline YouTube & $500 \mathrm{kbps}$ & 4.5 & Good & Perceptible but not annoying \\
\hline $\begin{array}{c}\text { Premium } \\
\text { YouTube }\end{array}$ & $1 \mathrm{Mbps}$ & 4.5 & & \\
\hline Netflix HQ & $5 \mathrm{Mbps}$ & 4.5 & & \\
\hline
\end{tabular}

\section{PERFORMANCE RESULTS}

Based on the simulation configuration described above, our approach and the other existing strategies for maximizing the SINR and the throughput estimation were executed in the controller every time a new user tried to join the network, or an active user needed a new flow with different QoS requirements. The achieved results are illustrated in Figs. 5, 6, and 7.

In detail, in Fig. 5 the upper and lower edges of the plotted boxes representing the data rate distribution are the 25 th and 75 th percentile of their values for 400 connected flows, while their median values are indicated by the central red lines. The values which we considered as outliers are indicated by red symbols. Accordingly, these results show how our FF-based approach allows a data rate assignment that depends on the data rate requirements illustrated in Table 4 . In fact, most of the assigned data rates are concentrated within the plotted box, i.e., between approximately $40 \mathrm{kbps}$ and $1 \mathrm{Mbps}$, while it is reduced the distribution of data rates higher than $1 \mathrm{Mbps}$. In case of Load aware-based and SINR-based solutions, the distribution of the assigned data rates higher than $1 \mathrm{Mbps}$ increases considerably with respect to our FF-based approach because they address best expected throughput and SINR, respectively, and do not take into account the data rate requirements. This results demonstrate how the proposed FF-based approach allows the best fairness in terms of the distribution of the data rates because it enables to assign the most suitable data rates to the requirements rather than the higher ones compared to the Load aware-based and SINR-based solutions.

The results shown in Fig. 5 have an implication of the satisfaction of wireless users as providing the required bit rate is an objective of our approach. This can be observed in Fig. 6, which illustrates the performance analysis in terms of the achieved satisfaction as a function of the number of the flows connecting to the network. This figure shows that the proposed FF-based scheme provides better flow satisfaction than the Load awarebased and SINR-based solutions. From this figure we can ob-

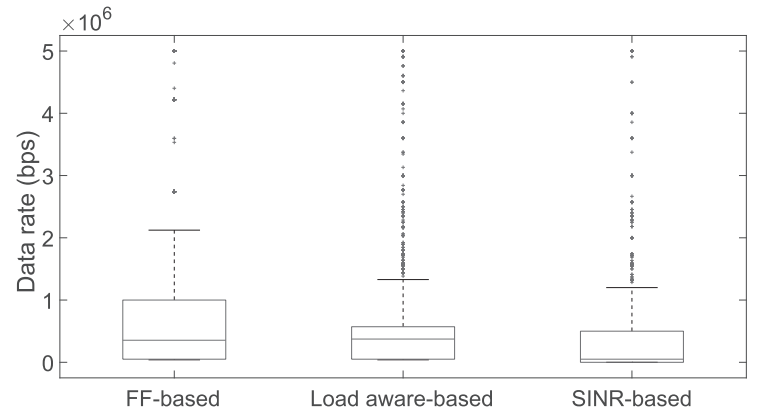

Fig. 5. Distribution of the data rates.

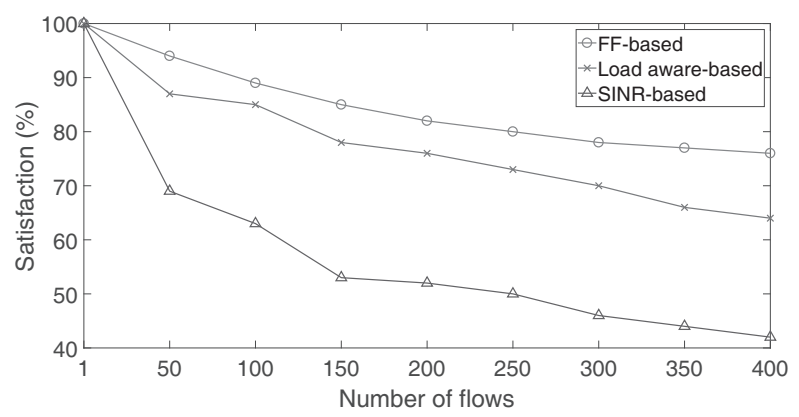

Fig. 6. Satisfaction percentage.

serve that when all the 400 flows are connected to the network our RAT selection scheme outperforms the Load aware-based strategy by around $16 \%$, and the SINR-based solution by around $45 \%$. This shows that our approach of RAT selection and the adopted FF metric reflect the satisfaction of the flows much better than other approaches that rely on other metrics.

Although satisfying wireless users' requirement is a main target of our solution, it is also necessary that this satisfaction is translated into an acceptable QoE. Fig. 7 shows the performance results in terms of the percentage of flows that reach at least a Good MOS for the three approaches. The left hand side of the figure illustrates the performance achieved in the case of Voice, while the right hand side shows the performance obtained in the case of Video. The figure illustrates that in the case of Voice, our FF-based scheme and the Load aware one guarantee a Good $M O S$ to all the flows connected to the network both improving on the SINR-based solution, which guarantees a Good MOS to only approximately the $68 \%$ of the flows. On the other hand, our RAT selection scheme outperforms both of the others in terms of the percentage of flows requiring a connection for a video streaming and reaching at least a Good MOS, the Load awarebased strategy by around $32 \%$, and the SINR-based solution by around $58 \%$.

In summary, from this performance analysis we can conclude that the proposed FF-based scheme gives the best fairness guaranteed by the suitability between the users' requirements in terms of bit rate and the selected RAT. It also allows us to achieve the best performance in terms of satisfaction and Good MOS compared to the state of the art. 


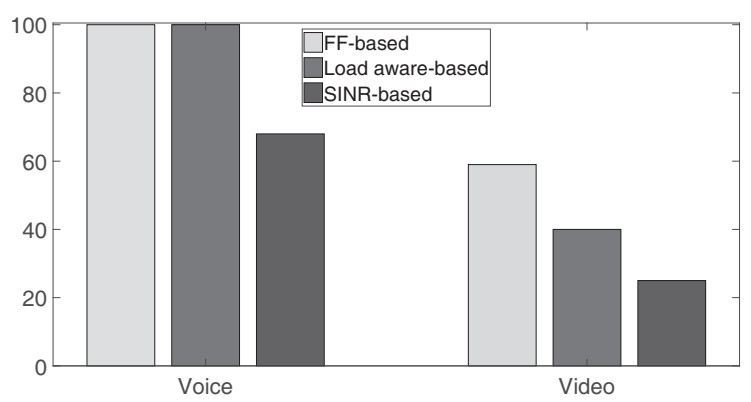

Fig. 7. Percentage of flows with at least Good MOS.

\section{CONCLUSION AND FUTURE WORK}

In this paper, we have proposed an SDN-based framework that assists wireless users to connect to the network that best satisfies their QoS requirements. The proposed framework implements an innovative RAT selection strategy that relies on a metric called FF. This metric takes into account the heterogeneity of the wireless users' requirements in terms of bit rate and the differing capabilities of the RAT technologies.

The RAT selection strategy is based on a potential game played by the SDN controller to find the most suitable distribution of the FFs between the flows and the RATs through the optimization of a network utility function. The use of SDN allows us to monitor and store all the required information for the computation of FFs between the users' requirements and each RAT and the network utility function on the controller. Therefore, the game can be played at the SDN controller exploiting its potentiality and overcoming its drawbacks in terms of scalability.

The proposed framework has been evaluated via simulation to enable its comparison against a RAT selection scheme based on 3GPP and 802.11 standards, and another solution considered in the literature based on the best throughput estimation. The evaluation results have demonstrated that our solution achieves significant improvements over both schemes in terms of the distribution of the data rate among the users, user satisfaction and QoE.

Motivated by the satisfactory results achieved through the SDN framework based on simulations, our future work will consider the implementation and assessment of our RAT selection framework in the Wi-5 real-time platform [8] in order to exploit its benefits in real HetNet environments.

\section{REFERENCES}

[1] Q. C. Li, H. Niu, A. T. Papathanassiou, and G. Wu, "5G network capacity: Key elements and technologies," IEEE Veh. Technol. Mag., vol. 9, no. 1, pp. 71-78, Mar. 2014.

[2] O. Galinina, A. Pyattaev, S. Andreev, M. Dohler, and Y. Koucheryavy, "5G multi-RAT LTE-WiFi ultra-dense small cells: Performance dynamics, architecture, and Trends," IEEE J. Select. Areas Commun., vol. 33, no. 6, pp. 1224-1240, June 2015.

[3] S. Andreev et al., "Intelligent access network selection in converged multiradio heterogeneous networks," IEEE Wireless Commun., vol. 21, no. 6, pp. 86-96, Dec. 2014.

[4] G. Judd and P. Steenkiste, "Fixing 802.11 access point selection," ACM SIGCOMM Computer Communication Review, vol. 32, no. 3, pp. 31, 2002.
[5] O. N. Fundation, "Software-defined networking: The new norm for networks," ONF White Paper, vol. 2, pp. 2-6, 2012.

[6] N. McKeown et al., "OpenFlow: Enabling innovation in campus networks," ACM SIGCOMM Computer Communication Review, vol. 38, no. 2, pp. 69-74, 2008.

[7] R. Riggio, T. Rasheed, and F. Granelli, "EmPOWER: A testbed for network function virtualization research and experimentation," in Proc. IEEE SDN4FNS, Trento, Italy, Nov. 2013.

[8] H2020 Wi-5 Project, "What to do With the Wi-Fi Wild West," [Online]. Available: http://www.wi5.eu/.

[9] F. Bouhafs, "Wi-5 initial architecture," Deliverable D2.4 of Wi-5 project, 2015.

[10] J. Saldana, "Specification of Smart AP solutions version 1," Deliverable D3.2 of Wi-5 project, 2015.

[11] S. Singh, H. S. Dhillon, and J. G. Andrews, "Offloading in heterogeneous networks: Modeling, analysis, and design insights," IEEE Trans. Wireless Commun., vol. 12, no. 5, pp. 2484-2497, May 2013.

[12] N. Wang, E. Hossain, and V. K. Bhargava, "Joint downlink cell association and bandwidth allocation for wireless backhauling in two-tier HetNets with large-scale antenna arrays," IEEE Trans. Wireless Commun., vol. 15, no. 5, pp. 3251-3268, May 2016.

[13] A. Roy, P. Chaporkar, and A. Karandikar, "An on-line radio access technology selection algorithm in an LTE-WiFi network," in Proc. IEEE WCNC, San Francisco, CA, USA, Mar. 2017.

[14] S. Maaloul, M. Afif, and S. Tabbane, "Handover decision in heterogeneous networks," in Proc. IEEE AINA, Crans-Montana, Switzerland, Mar. 2016.

[15] A. Awad, A. Mohamed, and C.-F. Chiasserini, "User-centric network selection in multi-RAT systems," in Proc. IEEE WCNCW, Doha, Qatar, Apr. 2016, pp. 97-102.

[16] L. Liu, L. Sun, and E. Ifeachor, "User-centric QoE-driven vertical handover framework in heterogeneous wireless networks," in Proc. IEEE WiMob, NY, USA, Oct. 2016, pp. 1-8.

[17] M. Behjati, J. P. Cosmas, R. Nilavalan, G. Araniti, and M. Condoluci, "Self-organising comprehensive handover strategy for multi-tier LTEAdvanced heterogeneous networks," IET Sci., Meas. \& Technol., vol. 8, no. 6, pp. 441-451, 2014.

[18] M. Selim, A. Kamal, K. Elsayed, H. Abd-El-Atty, and M. Alnuem, "A novel approach for back-haul self healing in 4G/5G HetNets," in Proc. IEEE ICC, London, UK, June 2015, pp. 3927-3932.

[19] L. Qiang, J. Li, Y. Ji, and C. Huang, "A novel software-defined networking approach for vertical handoff in heterogeneous wireless networks," Wireless Commun. Mobile Comput., vol. 16, no. 15, pp. 2374-2389, 2016.

[20] Z. Arslan, M. Erel, Y. Özcevik, and B. Canberk, "SDoff: A softwaredefined offloading controller for heterogeneous networks," in Proc. IEEE WCNC, Istanbul, Turkey, Apr. 2014, pp. 2827-2832.

[21] S. Sun, L. Gong, B. Rong, and K. Lu, "An intelligent SDN framework for 5G heterogeneous networks," IEEE Commun. Mag., vol. 53, no. 11 pp. 142-147, 2015.

[22] J. Zhang, W. Xie, and F. Yang, "An architecture for 5G mobile network based on SDN and NFV," in Proc. ICWMMN, Beijing, China, Apr. 2015, pp. 87-92.

[23] A. Raschellà, F. Bouhafs, M. Seyedebrahimi, M. Mackay, and Q. Shi, "A centralized framework for smart access point selection based on the fittingness factor," in Proc. ICT,Thessaloniki, Greece, May 2016, pp. 1-5.

[24] A. Raschella, F. Bouhafs, M. Seyedebrahimi, M. Mackay, and Q. Shi, "Quality of service oriented access point selection framework for large Wi-Fi networks," IEEE Trans. Network Service Manage., vol. 14, no. 2 , pp. 441-455, June 2017.

[25] M. Seyedebrahimi, F. Bouhafs, A. Raschellà, M. Mackay, and Q. Shi, "SDN-based channel assignment algorithm for interference management in dense Wi-Fi networks," in Proc. EuCNC, Athens, Greece, June 2016, pp. 128-132.

[26] M. Seyedebrahimi, et al., "A centralised Wi-Fi management framework for D2D communications in dense Wi-Fi networks," in Proc. IEEE CSCN, Berlin, Germany, Oct. 2016, pp. 1-6.

[27] M. Seyedebrahimi, F. Bouhafs, A. Raschella, M. Mackay, and Q. Shi, "Fine-grained radio resource management to control interference in dense Wi-Fi networks," in Proc. IEEE WCNC, San Francisco, CA, USA, Mar. 2017, pp. 1-6.

[28] R. Wallner and R. Cannistra, "An SDN approach: Quality of service using big switch's floodlight open-source controller," in Proc. Asia-Pacific Advanced Network, vol. 35, pp. 14-19, 2013.

[29] F. Rodríguez-Teja, C. Martinez-Cagnazzo, and E. G. Castro, "Bayesian classification: Methodology for network traffic classification combination," in Proc. ACM IWCMC, NY, USA, June 2010, pp. 769-773.

[30] T. T. Nguyen, G. Armitage, P. Branch, and S. Zander, "Timely and continuous machine-learning-based classification for interactive IP traffic," IEEE/ACM Trans. Netw., vol. 20, no. 6, pp. 1880-1894, Dec. 2012. 
[31] J. Pérez-Romero, O. Sallent, and R. Agustí, "A novel metric for contextaware RAT selection in wireless multi-access systems," in Proc. IEEE ICC, Glasgow, UK, June 2007, pp. 5622-5627.

[32] S. Sesia, M. Baker, and I. Toufik, LTE-the UMTS Long Term Evolution: From theory to practice. John Wiley \& Sons, 2011.

[33] A. Chiumento, S. Pollin, C. Desset, L. Van der Perre, and R. Lauwereins, "Scalable HetNet interference management and the impact of limited channel state information," EURASIP J. Wireless Commun. Netw., vol. 2015, no. 1, pp. 1-11, Mar. 2015.

[34] G. Araniti, M. Condoluci, L. Militano, and A. Iera, "Adaptive resource allocation to multicast services in LTE systems," IEEE Trans. Broadcast., vol. 59, no. 4, pp. 658-664, Dec. 2013.

[35] L. Badia, M. Lindstrom, J. Zander, and M. Zorzi, "Demand and pricing effects on the radio resource allocation of multimedia communication systems," in Proc. IEEE GLOBECOM, San Francisco, CA, USA, Dec. 2003, pp. 4116-4121.

[36] A. Raschellà, J. Pérez-Romero, O. Sallent, and A. Umbert, "On the use of POMDP for spectrum selection in cognitive radio networks," in Proc. IEEE CROWNCOM, , 2013, pp. 19-24.

[37] D. Monderer and L. S. Shapley, "Potential games," Games and Economic Behavior, vol. 14, no. 1, pp. 124-143, May 1996.

[38] J. Ortín, J. R. Gállego, and M. Canales, "Joint cell selection and resource allocation games with backhaul constraints," Pervasive and Mobile Comput., vol. 35, pp. 125-145, Feb. 2017.

[39] 3GPP, “3GPP TR 36.819,” http://www.wi5.eu/, 2012.

[40] S. F. Yunas, M. Valkama, and J. Niemelä, "Spectral and energy efficiency of ultra-dense networks under different deployment strategies," IEEE Commun. Mag., vol. 53, no. 1, pp. 90-100, Jan. 2015.

[41] K. Piamrat, A. Ksentini, C. Viho, and J.-M. Bonnin, "QoE-aware admission control for multimedia applications in IEEE 802.11 wireless networks," in Proc. IEEE VTC, Calgary, BC, Canada, Sept. 2008, pp. 1-5.

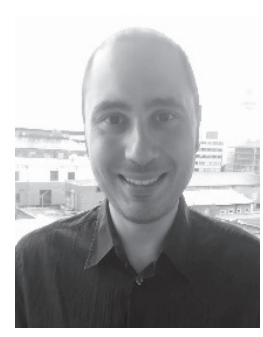

Alessandro Raschellà received the M.Sc. in Telecommunications Engineering from the University Mediterranea of Reggio Calabria (UNIRC), Italy in 2007 and the $\mathrm{Ph} . \mathrm{D}$. degree in Wireless Communications from the Universitat Politècnica de Catalunya (UPC), Barcelona, Spain in 2015. From 2007 to 2009, he was a Research Assistant with UNIRC. He is currently a Postdoctoral Research Associate with Liverpool John Moores University (LJMU), UK. His research interests currently include dynamic spectrum access and management, wireless networks optimization, cognitive radio networks, and HetNets.

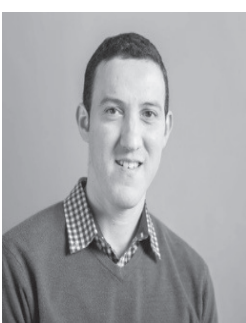

Faycal Bouhafs received a Computer Engineering degree in 1999, and Master's degree in Computer Science in 2002, and Ph.D. degree in 2007. Between 2007 and 2009 he worked as a research assistant with the University of Edinburgh, before joining Liverpool John Moores University as Senior Lecturer in Networking. His research interests include spectrum management in wireless networks and HetNets. He is currently the Technical Lead for the H2020 project Wi-5.

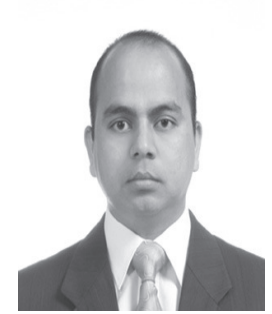

Deepak G.C. received Ph.D. degree in spectrum sharing system in cognitive radio networks in 2017 from Lancaster University, UK. He then joined Liverpool John Moores University (LJMU), UK as a Research Associate with $\mathrm{H} 2020$ project Wi-5. He is currently with Department of Computer Science and Mathematics at Kingston University, UK as a Research Associate. His research interests include dynamic resource allocation, spectrum sensing, Internet-of-Things, D2D and $\mathrm{M} 2 \mathrm{M}$ communication, massive-MIMO, and 5G.

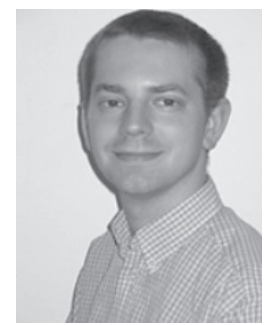

Michael Mackay received a Ph.D. degree in IPv6 transition management from Lancaster University in 2005 and has been a Senior Lecturer with the Department of Computer Science at Liverpool John Moores University since 2010 . His main research areas currently include cloud networking, IoT and smart applications, and wireless spectrum management. 